\title{
VARIAÇÕES ESTRUTURAIS E CARACTERÍSTICAS EDÁFICAS EM DIFERENTES ESTÁDIOS SUCESSIONAIS DE FLORESTA CILIAR DE TABULEIRO, ES ${ }^{1}$
}

\author{
Luiz Fernando Silva Magnago², Marcelo Simonelli³, Sebastião Venâncio Martins ${ }^{4}$, \\ Fabio Antonio Ribeiro Matos²e Valdir Geraldo Demuner ${ }^{5}$
}

\begin{abstract}
RESUMO - Este estudo teve como objetivo analisar a comunidade arbórea em três estádios sucessionais florestais ciliares na Área de Proteção Ambiental Lagoa de Jacunem, no Município da Serra, ES. Para a caracterização estrutural foram empregadas 60 parcelas de $10 \mathrm{~m}^{2}$, totalizando 0,6 ha amostrado. As parcelas foram distribuídas equitativamente entre cada estádio sucessional, onde foram incluídos os indivíduos com CAP $\geq 15$ cm, a 1,30 m do solo. Em cada estádio também foram coletadas amostras compostas de solo para posterior análise química. As áreas apresentaram solos distróficos, sendo por isso pobre em nutrientes e com acidez elevada. Foi amostrado um total de 851 indivíduos, distribuídos em 79 espécies. A diversidade (H’) de espécies foi aumentando gradativamente seus valores, sendo 2,70 no estádio inicial, 2,88 no estádio médio e 2,96 no estádio avançado, respectivamente. A equabilidade J' foi de 0,77 nos estádios inicial e médio e 0,73 no estádio avançado. A densidade decresce significativamente (ANOVA; $\mathrm{F}=6,55 ; \mathrm{P}<0,01$ ) em direção aos serais mais avançados. Os valores de área basal total variaram significativamente entre os estádios sucessionais (ANOVA; F = 24,87; P<0,0000001). Esses resultados evidenciaram características estruturais e diversidades diferenciadas de acordo com o estádio de sucessão em que cada fragmento se encontrava.
\end{abstract}

Palavras-chave: Floresta Atlântica, Sucessão florestal e Solos distróficos.

\section{STRUCTURAL CHANGES AND EDAPHIC CHARACTERISTICS IN DIFFERENT SUCCESSION STAGES OF TABULEIRO RIPARIAN FOREST, ES}

\begin{abstract}
The objective of this work was to analyze tree community of three riparian successional forest stages in Environment Protection Area of Lagoa do Jacunem, in Serra, Espírito Santo. For the structural characterization, 60 plots of $10 \mathrm{~m}^{2}$ were used, totalizing 0.6 ha of the sampled. The plots were equally distributed among each succession stage, where it was included specimens with $C B H \geq 15 \mathrm{~cm}$ at $1.30 \mathrm{~m}$ from the soil. In each stage, it was also collected composed samples of soil for posterior chemical analyses. The areas presented dystrophic soils and therefore low in nutrients and high acidity. It was sampled a total of 851 individuals, distributed in 79 species. The diversity ( $H^{\prime}$ ) of species was gradually increasing its values, being 2.70 in the initial stage, 2.88 in the middle stage, and 2.96 in advanced stage, respectively. The equability J'was 0.77 in the initial and middle stages, and 0.73 in the advanced stage. The density decreased significantly (ANOVA; $F=6.55 ; P<0.01)$ towards more advanced seral. The values of total basal area varied significantly among successional stages (ANOVA; $F=24.87 ; P<0.0000001$ ). These results evidenced the structural characteristics and differentiated diversity according to the stage of succession where each fragment was.
\end{abstract}

Keywords: Atlantica Forest, Forest succession and Dystrophic soils.

\footnotetext{
${ }^{1}$ Recebido em 23.06.2009 e aceito para publicação em 14.04.2011.

${ }^{2}$ Programa de Pós-Graduação em Botânica pela Universidade Federal de Viçosa, UFV, Brasil. E-mail: <luiz_fsm@hotmail.com> e $<$ fabiomatos82@gmail.com>.

${ }^{3}$ Instituto Federal de Educação, Ciência e Tecnologia do Espírito Santo, IFES, Brasil. E-mail: <msimonelli@ides.edu.br> e<mailto:msimonelli@ides.edu.br>.

${ }^{4}$ Departamento de Engenharia Florestal da Universidade Federal de Viçosa, UFV, Brasil. E-mail: <venancio@ufv.br>.

${ }^{5}$ Pesquisador voluntário do Museu de Biologia Prof. Mello Leitão, MBML, Brasil. E-mail: <vgdemu@yahoo.com.br>.
} 


\section{INTRODUÇÃO}

A Mata Atlântica é a segunda maior floresta pluvial tropical do continente americano, estendendo-se originalmente de forma contínua ao longo da costa brasileira, adentrando até o leste do Paraguai e nordeste da Argentina em sua porção sul, apresentando uma alta heterogeneidade em sua composição de espécies e fitofisionomias (TABARELLI et al., 2005). Inseridas no domínio da Mata Atlântica do Espírito Santo, estão as florestas ciliares, que, segundo Magnago et al. (2007), possuem condições ecológicas próprias que são empregadas pelo regime hídrico, sendo bastante heterogêneas quanto à diversidade de espécies e ambientes, mas, devido à sua exploração para uso agrícola, encontram-se em nível critico de conservação nesse Estado.

Através dos processos de regeneração natural, áreas florestais com intensa degradação podem se recuperar (MARTINS, 2007). O termo sucessão vegetacional pode ser entendido como os processos gerais de mudanças na vegetação, que ocorrem em uma mesma área ao longo do tempo, consistindo em alterações na composição e diversidade de espécies, exclusão competitiva e aumento da complexidade estrutural da vegetação (MÜLLER-DOMBOIS; ELLENBERG, 1974). Essas mudanças na vegetação são também acompanhadas por alterações no habitat, como diminuição da intensidade luminosa, incremento de biomassa e disponibilidade de nutrientes no solo (TILMAN, 1988; BROWN; LUGO, 1990; GUARIGUATA; OSTERTAG, 2001).

Estudos fitossociológicos com ênfase na caracterização dos processos sucessionais podem ser desenvolvidos através de análises em uma mesma área, empregando-se o método de parcelas permanentes, ou podem ser conduzidos em diferentes trechos de florestas que se apresentam em cronossequências sucessionais diferentes (MÜLLER-DOMBOIS; ELLENBERG, 1974). Pesquisas que utilizam o método de cronossequência apresentam resultados satisfatórios com maior rapidez, diminuem os custos do estudo e permitem inferir sobre os padrões de mudanças na estrutura e na composição florística que ocorrem em uma área (ARAÚJO et al., 2005).

Dessa forma, os estudos fitossociológicos de cronossequência são de suma importância na geração de informações estratégicas para a implantação de programas de recuperação ambiental, principalmente quando se utilizam espécies nativas (VILELA et al., 1993), já que diversos modelos de plantios para a restauração florestal são baseados nos processos dinâmicos sucessionais de florestas nativas (MARTINS, 2007).

Mesmo com essa importância, não há estudos sobre variações estruturais em diferentes estádios sucessionais para esse Estado, mas estudos fitossociológicos foram feitos por Peixoto e Gentry (1990), López (1996), Rolim e Nascimento (1997), Simonelli et al. (2008), Jesus e Rolim (2005), restritos à Reserva da Vale do Rio Doce. Com um enfoque para a estrutura de florestas ciliares do Estado, há apenas o trabalho de Thomaz (1996) para a Mata Atlântica de Encosta. Recentemente, Paula et al. (2009) realizaram um estudo com florestas de Tabuleiro no entorno da Lagoa Juparanã em Linhares/ES.

A fim de contribuir com o conhecimento sobre a estrutura e as características sucessionais de florestas ciliares do Espírito Santo, este trabalho tem como objetivo estudar ecologicamente três estádios sucessionais de florestas ciliares de Tabuleiro na Área de Proteção Ambiental da Lagoa de Jacunem, município da Serra, Espírito Santo.

\section{MATERIAL DE MÉTODOS}

A Área de Proteção Ambiental (APA) da Lagoa de Jacunem localiza-se no município da Serra, na região central espírito-santense, entre as coordenadas 2009’40”'S e 40¹3’08’'W. Segundo IBGE (1987), a região onde está localizada a APA se encontra no domínio de Floresta Ombrófila Densa de Terras Baixas. Jesus e Rolim (2005) denominam esse ecossistema de Floresta Atlântica de Tabuleiro, localizado em terrenos originados no Terciário, sendo o solo pertencente ao grupo Barreiras.

Para análise fitossociológica, foi utilizado o método de parcelas (MÜLLER-DOMBOIS; ELLENBERG, 1974), sendo estabelecidas 60 parcelas de $10 \mathrm{~m}^{2}$, distribuídas de maneira uniforme nos três estádios sucessionais estudados (estádio inicial, médio e avançado), totalizando 0,6 ha amostrado. As parcelas foram alocadas paralelamente às margens da Lagoa de Jacunem (posicionadas a uma distância média de $10 \mathrm{~m}$ da margem), com intervalos de 10m. Foram incluídas na amostragem todas as plantas, inclusive as mortas em pé, com circunferência à altura do peito $(\mathrm{CAP}) \geq 15 \mathrm{~cm}$ a $1,30 \mathrm{~m}$ do solo. 
Para análise química do solo das florestas em estádio inicial, médio e avançado, foram coletadas 20 amostras simples de solo em cada estádio, coletadas de 0-10 cm de profundidade, formando uma amostra composta para cada estádio. As análises foram feitas no laboratório do Departamento de Solos da Universidade Federal de Viçosa. A avaliação dos resultados foi feita a partir das recomendações para interpretação de análise de solos de Alvarez et al. (1999).

Os parâmetros fitossociológicos analisados foram frequência, densidade e dominância absolutas e seus respectivos valores relativos, sendo também calculados a área basal e valor de importância (MÜLLER-DOMBOIS; ELLENBERG, 1974). O índice de diversidade de Shannon ( $H^{\prime}$ ) e o de equabilidade de Pielou (J') foram calculados conforme Brower e Zar (1984). As análises fitossociológicas foram realizadas no programa Fitopac 1 (SHEPHERD, 1994).

As espécies foram determinadas a partir de consultas ao Herbário CVRD da Vale do Rio Doce e Herbário VIES da Universidade Federal do Espírito Santo, bem como com auxílio de literaturas especializadas e envio para especialistas. As plantas encontradas em estádio fértil foram depositadas no Herbário MBML do Museu de Biologia Prof. Mello Leitão.

As diferenças nos valores de densidade, área basal e altura entre as florestas analisadas foram testadas com auxílio do teste de variância paramétrica ANOVA one way, sendo posteriormente empregado o teste de Tukey para verificar as áreas em que as diferenças foram significativas. Os resultados desses testes foram considerados significativos a 5\% de probabilidade. Para a realização dos testes estatísticos propostos, os dados foram analisados quanto à sua normalidade através do teste de Shapiro-Wilk, e para o teste de homocedasticidade das variâncias dos dados foi utilizado o teste de Levene.

\section{RESULTADOS}

A análise química do solo dos três estádios sucessionais indicou a saturação por bases (V) baixa no estádio inicial e médio e muito baixa no estádio avançado, sendo os teores de macronutrientes ( $\mathrm{P}, \mathrm{K}$, Ca e Mg) baixos nos três estádios (Tabela 1). Os teores de $\mathrm{Al}$ aumentam gradativamente do estádio inicial para o avançado, variando de baixo a bom. Os solos dos três estádios apresentaram-se ácidos, sendo o inicial e o médio com acidez elevada e o avançado com acidez muito elevada. De acordo com Alvarez et al. (1999), o solo do estádio avançado apresentou bons teores de matéria orgânica, e os demais estádios apresentaram teores médios.

Nos três estádios sucessionais, foram amostrados 851 indivíduos, onde foi levantado um total de 79 espécies. Em uma comparação entre os três estádios estudados, o número de indivíduos levantados e a

Tabela 1 - Resultados das análises químicas dos solos nos três estádios sucessionais da APA da Lagoa de Jacunem. EI = Estádio inicial; EM = Estádio médio; e EA = Estádio avançado.

Table 1 - Results of the chemical analysis of soils in the three succession stages of Lagoa de Jacunem EPA. EI = Initial stage $; E M=$ Middle stage $;$ and $E A=$ Advanced stage .

\begin{tabular}{|c|c|c|c|c|}
\hline Características químicas & Unidades & EI & EM & EA \\
\hline$\overline{\mathrm{pH}}$ & $\mathrm{H}_{2} \mathrm{O}$ & 5,05 & 4,71 & 4,31 \\
\hline $\mathrm{P}$ & $\mathrm{mg} / \mathrm{dm}^{3}$ & 1,4 & 2,5 & 1,4 \\
\hline $\mathrm{K}$ & $\mathrm{mg} / \mathrm{dm}^{3}$ & 22 & 34 & 35 \\
\hline $\mathrm{Ca}^{2+}$ & $\mathrm{cmol} / \mathrm{dm}^{3}$ & 1,2 & 1,19 & 0,77 \\
\hline $\mathrm{Mg}^{2+}$ & $\mathrm{cmol} / \mathrm{dm}^{3}$ & 0,66 & 0,42 & 0,33 \\
\hline $\mathrm{Al}^{+3}$ & $\mathrm{cmol} / \mathrm{dm}^{3}$ & 0,48 & 0,58 & 1,25 \\
\hline Acidez potencial $(\mathrm{H}+\mathrm{Al})$ & $\mathrm{cmol} / \mathrm{dm}^{3}$ & 5,4 & 4,6 & 9,2 \\
\hline Soma de bases (SB) & $\mathrm{cmol} / \mathrm{dm}^{3}$ & 1,92 & 1,7 & 1,15 \\
\hline CTC efetiva (t) & $\mathrm{cmol} / \mathrm{dm}^{3}$ & 2,4 & 2,28 & 2,4 \\
\hline CTC pH 7 (T) & $\mathrm{cmol} / \mathrm{dm}^{3}$ & 7,32 & 6,3 & 10,35 \\
\hline Saturação por bases (V) & $\%$ & 26,2 & 27 & 11,1 \\
\hline Saturação por $\mathrm{Al}^{+3}(\mathrm{~m})$ & $\%$ & 20 & 25,4 & 52,1 \\
\hline Matéria orgânica (MO) & dag/kg & 3,82 & 3,03 & 5,66 \\
\hline P-rem & $\mathrm{mg} / \mathrm{L}$ & 39,6 & 44,9 & 32 \\
\hline
\end{tabular}


Tabela 2 - Dados da estrutura da vegetação nos três estádios sucessionais da APA da Lagoa de Jacunem. EI = Estádio inicial; EM = Estádio médio; EA = Estádio avançado de sucessão; NI = Número de indivíduos; DT = Densidade total; $\mathrm{AB}=$ Área Basal; Alt = Altura; e H’= Diversidade de Shannon.

Table 2 - Data of vegetation structure in three succession stages of the Lagoa de Jacunem EPA. EI = Initial stage; $E M=$ Middle stage $; A A=$ Advanced stage. $N I=$ Number of individuals $; D T=$ total density; $A B=$ Basal area; Alt $=$ Height; and $H^{\prime}=$ Shannon diversity.

\begin{tabular}{|c|c|c|c|c|c|c|c|c|c|}
\hline \multirow{2}{*}{$\begin{array}{l}\text { Estádios } \\
\text { sucessionais }\end{array}$} & \multicolumn{6}{|c|}{ Árvores vivas } & \multicolumn{3}{|c|}{ Árvores mortas } \\
\hline & NI & DT(ind/ha) & $\mathbf{A B}\left(\mathbf{m}^{2} / \mathbf{h a}\right)$ & Alt. & média(m) & $H^{\prime}($ nats/ind) & NI & DT(ind/ha) & $A B\left(m^{2} / h a\right)$ \\
\hline EI & $314 \mathrm{a}$ & 1570 a & 12,766 a & & 4,73 a & 2,70 & 22 & 110 & 0,53 \\
\hline EM & $283 b$ & $1415 \mathrm{~b}$ & $32,184 \mathrm{~b}$ & & $9,34 \mathrm{~b}$ & 2,88 & 31 & 155 & 1,64 \\
\hline EA & $254 \mathrm{~b}$ & $1270 \mathrm{~b}$ & $22,35 \mathrm{c}$ & & $8,6 \mathrm{~b}$ & 2,96 & 12 & 60 & 0,69 \\
\hline
\end{tabular}

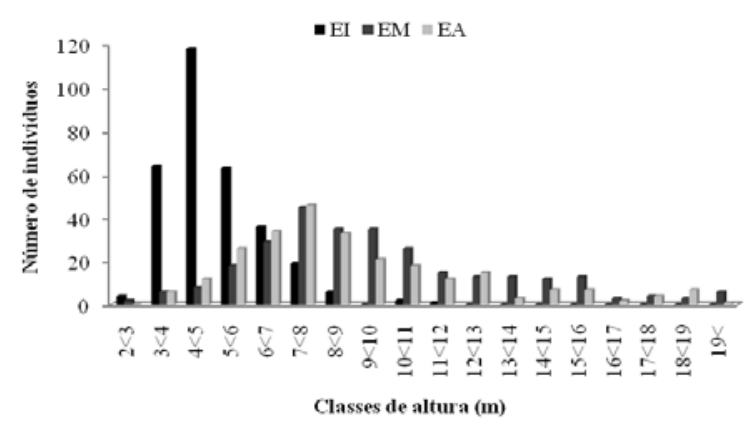

Figura 1 - Distribuição do número de indivíduos em classes de altura das espécies amostradas nos três estádios sucessionais da APA da Lagoa de Jacunem. EI = Estádio inicial; EM = Estádio médio; e EA = Estádio avançado de sucessão.

Figure 1-Distribution of the number of individuals in height classes of the species sampled in three succession stages of the Lagoa de Jacunem EPA. EI = Initial stage $; E M=$ Middle stage $;$ and $E A=$ Advanced stage.

densidade de indivíduos por hectare apresentou uma ordem decrescente em direção ao avanço sucessional (Tabela 2), sendo a variação estatisticamente significativa (ANOVA; $\mathrm{F}=7,41 ; \mathrm{P}<0,001$ ), porém o teste de Tukey evidenciou diferenças entre o estádio avançado com o estádio inicial $(\mathrm{P}<0,001)$, e este último com o estádio médio $(\mathrm{P}<0,05)$, portanto não foram encontradas diferenças significativas entre o estádio avançado com o médio $(\mathrm{P}<0,49)$.

A altura média das espécies amostradas nos estádios inicial, médio e avançado foram de 4,73m, 9,34m e 8,60m, respectivamente (Figura 1), sendo a variação encontrada entre os estádios significativa (ANOVA; F=79,95; $\mathrm{P}<0,0001)$. O teste de Tukey revelou que a altura do estádio inicial é estatisticamente diferente daquela dos demais estádios $(\mathrm{P}<0,0001)$, porém as alturas do estádio avançado e médio não foram significativamente diferentes $(\mathrm{P}<0,26)$.

O estádio inicial não apresentou sub-bosque bem delimitado, e as espécies que caracterizam o dossel foram Gochnatia polymorpha, Coccoloca alnifolia e Byrsonima sericea, onde foram encontradas algumas espécies emergentes, como Protium heptaphyllum e Tapirira guianensis.

Já o estádio médio e o avançado apresentaram melhor distribuição em classes de altura. As espécies que caracterizaram o dossel dessas florestas foram Protium heptaphyllum, Tapirira guianensis, Myrcia fallax e Miconia prasina. Entre as emergentes estão Pseudopiptadenia contorta e Apuleia leiocarpa. Esses estádios apresentaram um sub-bosque bem delimitado, estando Guapira opposita, Eugenia punicifolia e Myrcine umbellata entre as espécies mais características desse estrato.

A análise de distribuição dos indivíduos amostrados nos três estádios sucessionais em classes diamétricas revelou um padrão em J-invertido, desta maneira apresentando a maior concentração de indivíduos nas classes de menor diâmetro, seguindo um decréscimo em direção às classes maiores (Figura 2). As classes diamétricas entre 4,8 e $10 \mathrm{~cm}$ e 10 e 15 cm congregam 55\% e $25 \%$, respectivamente, dos indivíduos no estádio inicial de sucessão. No estádio médio, os indivíduos se distribuem de maneira mais gradual entre as duas primeiras classes, sendo $39 \%$ entre 4,8 e $10 \mathrm{~cm}$ e $22 \%$ entre 10 e $15 \mathrm{~cm}$. No estádio avançado, os indivíduos apresentam 43\% e 23\% nas classes entre 4,8 e $10 \mathrm{~cm}$ e 10 e $15 \mathrm{~cm}$, respectivamente. 


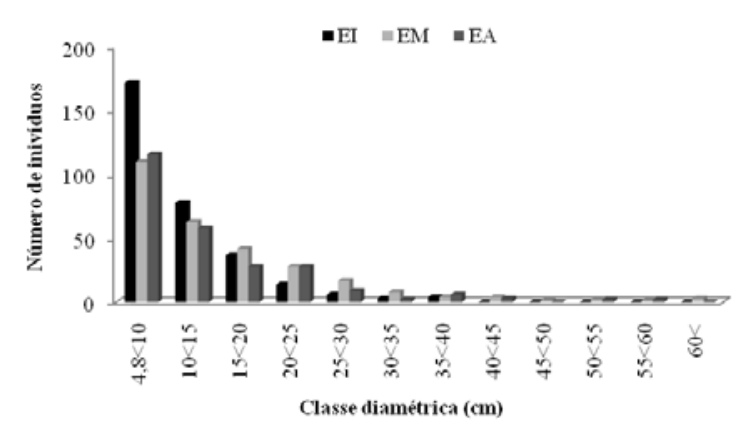

Figura 2 - Distribuição do número de indivíduos em classes diamétricas das espécies amostradas nos três estádios sucessionais da APA da Lagoa de Jacunem. EI = Estádio inicial; EM = Estádio médio; e EA = Estádio avançado de sucessão.

Figure 2-Distribution of the number of individuals in diameter classes of the species sampled in three succession stages of Lagoa de Jacunem EPA. EI = Initial stage; $E M=$ Middle stage $;$ and $E A=$ Advanced stage.

A diversidade ( $\left.\mathrm{H}^{\prime}\right)$ encontrada nos estádios analisados aumentou de acordo com o avanço da sucessão. No estádio inicial, a diversidade foi de 2,70nats/ indivíduo. No estádio médio, esta foi de 2,88nats/indivíduo e, no estádio avançado, o valor encontrado foi 2,96nats/ indivíduo. A equabilidade $J$ ’ foi igual nos estádios inicial e médio $(0,77)$, tendo uma pequena diminuição no estádio avançado $(0,72)$.

O maior valor para área basal foi no estádio médio com $32,18 \mathrm{~m}^{2} / \mathrm{ha}$. No estádio inicial, este valor foi $12,77 \mathrm{~m}^{2} /$ ha e, no estádio avançado, foi de $22,35 \mathrm{~m}^{2} / \mathrm{ha}$. Essas variações mostraram-se estatisticamente significativas (ANOVA; $F=24,87 ; \mathrm{P}<0,0000001$ ), sendo encontrados resultados significativamente diferentes entre todos os estádios estudados (Tukey; $\mathrm{P}<0,01$ ).

No estádio inicial, as espécies com maior valor de importância (VI) foram Gochnatia polymorpha $(76,28)$, seguidas de Eschweilera ovata $(42,42)$, Protium heptaphyllum (23,82), Pera glabrata $(13,75)$ e Tapirira guianensis $(13,23)$ (Tabela 3). No estádio médio, Protium heptaphyllum $(57,43)$, Eschweilera ovata $(44,83)$, Tapirira guianensis $(16,25)$, Ocotea notata $(15,57)$ e Gochnatia polymorpha $(15,21)$ foram as mais importantes (Tabela 4). Na floresta em estádio avançado, as mais importantes foram Eschweilera ovata (59,29), Protium heptaphyllum $(58,69)$, Thyrsodium schomburgkianum $(18,22)$, Miconia prasina $(14,58)$ e Myrcia fallax $(12,92)$ (Tabela 5).
As árvores mortas em pé tiveram um aumento no número de indivíduos e densidade total do estádio inicial para o estádio médio de sucessão, seguido de um decréscimo em direção ao estádio avançado (Tabela 2). A área basal também alcançou os maiores valores no estádio médio. O estádio avançando apresentou menos indivíduos mortos em pé que o estádio inicial, mas apresentou maior área basal.

A análise dos parâmetros estruturais de Eschweilera ovata denota maior desenvolvimento dos indivíduos da espécie em direção aos trechos em estádio médio e avançado de sucessão (Figura 3). Myrcia fallax e Protium heptaphyllum, que são secundárias iniciais, também apresentaram maior desenvolvimento em direção aos estádios mais avançados. As secundárias iniciais, Pera glabrata e Tapirira guianensis, apresentaram seus maiores valores de importância e área basal no estádio médio, seguidos de decréscimo em direção ao estádio avançado, tendo inversão apenas para os valores de densidade relativa. Gochnatia polymorpha apresentou redução no valor de importância, da área basal e densidade relativa em direção aos estádios sucessionais mais avançados.

\section{DISCUSSÃO}

Os solos dos três estádios são caracterizados como distróficos e ácidos e, portanto, apenas a fertilidade química do solo não é suficiente para explicar as diferenças estruturais entre eles. O histórico de regeneração florestal de cada estádio deve ser o principal fator envolvido nas diferenças estruturais apresentadas a seguir, já que as áreas são adjacentes, sob o mesmo tipo climático, exposição do terreno, características semelhantes de solo e com entorno também semelhante.

Os resultados encontrados na análise química do solo nos estádios estudados provavelmente não refletem as características sucessionais das florestas, já que, segundo Jacomine (2001), nas matas ciliares em geral, há a predominância de solos com alta matéria orgânica, solos ácidos a fortemente ácidos, pobres em macronutrientes, com baixa saturação por bases e elevada saturação de alumínio extraível. Em contrapartida, do ponto de vista da recuperação de áreas degradadas, é interessante que solos pobres e ácidos venham a subsidiar o estabelecimento de ecossistemas florestais bem desenvolvidos, como os encontrados na APA Lagoa de Jacunem.

Revista Árvore, Viçosa-MG, v.35, n.3, p.445-456, 2011 
Tabela 3 - Parâmetros fitossociológicos das espécies amostradas no estádio inicial da APA da Lagoa de Jacunem em ordem decrescente de VI. NI = Número de indivíduos; AB = Área basal; FA = Frequência absoluta; DA = Densidade absoluta; DoA = Dominância absoluta; FR = Frequência relativa; $\mathrm{DR}$ = Densidade relativa; DoR = Dominância relativa; and VI = Valor de importância.

Table 3 - Phytosociological parameters of the species sampled in the initial stage of the Lagoa de Jacunem EPA in decreasing order of IV. NI = number of individuals; $A B=$ basal area; $F A=$ Absolute frequence; $F R=$ Relative frequence; $D A=$ absolute density $; D R=$ Relative density $; D o A=$ Absolute dominance $; D o R=$ Relative dominance $;$ and $V I=$ importance value.

\begin{tabular}{|c|c|c|c|c|c|c|c|c|c|}
\hline Espécie & NI & AB & FA & DA & DoA & FR & DR & DoR & VI \\
\hline Gochnatia polymorpha (Less.) Cabrera. & 75 & 0,921 & 100,00 & 416,70 & 5,115 & 12,33 & 23,89 & 40,07 & 76,28 \\
\hline Eschweilera ovata (Cambess) Miers. & 63 & 0,262 & 88,89 & 350,00 & 1,455 & 10,96 & 20,06 & 11,40 & 42,42 \\
\hline Protium heptaphyllum (Aubl.) Marchand & 28 & 0,154 & 66,67 & 155,60 & 0,854 & 8,22 & 8,92 & 6,69 & 23,82 \\
\hline Pera glabrata Baill. & 13 & 0,079 & 50,00 & 72,20 & 0,440 & 6,16 & 4,14 & 3,44 & 13,75 \\
\hline Tapirira guianensis Aubl. & 11 & 0,145 & 27,78 & 61,10 & 0,805 & 3,42 & 3,50 & 6,31 & 13,23 \\
\hline Coccoloba alnifolia Casar. & 15 & 0,061 & 44,44 & 83,30 & 0,340 & 5,48 & 4,78 & 2,67 & 12,92 \\
\hline Byrsonima sericea DC. & 12 & 0,071 & 38,89 & 66,70 & 0,394 & 4,79 & 3,82 & 3,09 & 11,70 \\
\hline Thyrsodium schomburgkianum Benth. & 11 & 0,056 & 38,89 & 61,10 & 0,311 & 4,79 & 3,50 & 2,43 & 10,73 \\
\hline Andira fraxinifolia Benth. & 8 & 0,064 & 33,33 & 44,40 & 0,354 & 4,11 & 2,55 & 2,77 & 9,43 \\
\hline Cupania zanthoxyloides Cambess. & 7 & 0,071 & 27,78 & 38,90 & 0,394 & 3,42 & 2,23 & 3,09 & 8,74 \\
\hline Jacaranda puberula Cham. & 10 & 0,031 & 27,78 & 55,60 & 0,174 & 3,42 & 3,18 & 1,36 & 7,97 \\
\hline Myrcia fallax (Rich.)DC. & 6 & 0,032 & 27,78 & 33,30 & 0,176 & 3,42 & 1,91 & 1,38 & 6,71 \\
\hline Eugenia punicifolia (Humb., Bonpl. \& Kunth) DC. & 6 & 0,017 & 27,78 & 33,30 & 0,095 & 3,42 & 1,91 & 0,74 & 6,08 \\
\hline Himatanthus phagedaenicus (Mart.)Woodson & 4 & 0,022 & 22,22 & 22,20 & 0,122 & 2,74 & 1,27 & 0,96 & 4,97 \\
\hline Miconia albicans (Sw.) Triana. & 4 & 0,012 & 22,22 & 22,20 & 0,065 & 2,74 & 1,27 & 0,51 & 4,52 \\
\hline Inga thibaudiana DC. & 4 & 0,036 & 11,11 & 22,20 & 0,199 & 1,37 & 1,27 & 1,56 & 4,21 \\
\hline Xylopia sericea A. St.-Hil. & 3 & 0,021 & 16,67 & 16,70 & 0,117 & 2,05 & 0,96 & 0,91 & 3,92 \\
\hline Pseudopiptadenia contorta (DC.) & 3 & 0,031 & 11,11 & 16,70 & 0,173 & 1,37 & 0,96 & 1,35 & 3,68 \\
\hline \multicolumn{10}{|l|}{ G.P.Lewis \& M.P.M.de Lima } \\
\hline Inga flagelliformis (Vell.Conc.)Martius. & 4 & 0,017 & 11,11 & 22,20 & 0,096 & 1,37 & 1,27 & 0,75 & 3,40 \\
\hline Psychotria carthagenensis Jacq. & 3 & 0,022 & 11,11 & 16,70 & 0,123 & 1,37 & 0,96 & 0,97 & 3,29 \\
\hline Matayba guianensis Aubl & 2 & 0,028 & 11,11 & 11,10 & 0,157 & 1,37 & 0,64 & 1,23 & 3,24 \\
\hline Inga laurina Willd. & 3 & 0,016 & 11,11 & 16,70 & 0,087 & 1,37 & 0,96 & 0,68 & 3,00 \\
\hline Myrsine umbellata Mart. & 3 & 0,025 & 5,56 & 16,70 & 0,137 & 0,68 & 0,96 & 1,07 & 2,71 \\
\hline Casearia sp. & 2 & 0,016 & 11,11 & 11,10 & 0,088 & 1,37 & 0,64 & 0,69 & 2,69 \\
\hline Licania sp. & 3 & 0,007 & 11,11 & 16,70 & 0,036 & 1,37 & 0,96 & 0,28 & 2,61 \\
\hline Vismia brasiliensis Choisy. & 2 & 0,004 & 11,11 & 11,10 & 0,022 & 1,37 & 0,64 & 0,17 & 2,18 \\
\hline Dictyoloma vandellianum Adr. Juss. & 1 & 0,024 & 5,56 & 5,60 & 0,134 & 0,68 & 0,32 & 1,05 & 2,05 \\
\hline Zanthoxylum rhoifolium Lam. & 1 & 0,016 & 5,56 & 5,60 & 0,088 & 0,68 & 0,32 & 0,69 & 1,69 \\
\hline Swartzia apetala Raddi & 2 & 0,006 & 5,56 & 11,10 & 0,031 & 0,68 & 0,64 & 0,25 & 1,57 \\
\hline Ocotea notata (Nees) Mez & 1 & 0,009 & 5,56 & 5,60 & 0,050 & 0,68 & 0,32 & 0,39 & 1,39 \\
\hline Eugenia rotundifolia Casar. & 1 & 0,008 & 5,56 & 5,60 & 0,046 & 0,68 & 0,32 & 0,36 & 1,36 \\
\hline Clusia spiritu-sanctensis G. Mariz \& Weinberg & 1 & 0,007 & 5,56 & 5,60 & 0,041 & 0,68 & 0,32 & 0,32 & 1,33 \\
\hline Chrysophyllum lucentifolium Cronquist. & 1 & 0,007 & 5,56 & 5,60 & 0,040 & 0,68 & 0,32 & 0,31 & 1,32 \\
\hline Schinus terebinthifolius Raddi & 1 & 0,002 & 5,56 & 5,60 & 0,010 & 0,68 & 0,32 & 0,08 & 1,08 \\
\hline
\end{tabular}

A diminuição da densidade de indivíduos, em sentido do estádio inicial para os mais avançados, reflete a redução de uma grande quantidade indivíduos pertencentes a classes menores de diâmetro, tendo por isso um aumento no desenvolvimento das florestas. Este processo, denominado autodesbate, tem sido constatado em diversas florestas à medida que a sucessão natural avança (OLIVEIRA-FILHO et al.,1994; TABARELLI; MANTOVANI,1999; PAULA et al., 2004).
O padrão de distribuição diamétrica em J-invertido encontrado neste estudo para os três estádios sucessionais é comum em florestas em regeneração (CAVALCANTE et al., 2000). O decréscimo gradual no número de indivíduos encontrados em direção às classes superiores de diâmetro, segundo Araújo et al. (2006), indica que a floresta é composta, em sua maioria, por populações jovens. Dessa forma, as florestas analisadas possuem um contingente de indivíduos regenerantes que podem subsidiar o avanço sucessional desta 
Tabela 4 - Parâmetros fitossociológicos das espécies amostradas no estádio médio da APA da Lagoa de Jacunem. NI = Número de indivíduos; AB = Área basal; FA = Frequência absoluta; DA = Densidade absoluta; DoA = Dominância absoluta; $\mathrm{FR}=$ Frequência relativa; $\mathrm{DR}=$ Densidade relativa; DoR = Dominância relativa; e VI = Valor de importância.

Table 4-Phytosociological parameters of the species sampled in the middle stage of Lagoa de Jacunem EPA. NI=number of individuals; $A B=$ basal area $F A=$ Absolute frequence $; F R=$ Relative frequence $D A=$ absolute density; $D R=$ Relative density $; D o A=$ Absolute dominance $; D o R=$ Relative dominance and $V I=$ importance value.

\begin{tabular}{|c|c|c|c|c|c|c|c|c|c|}
\hline Espécie & NI & $\mathbf{A B}$ & FA & DA & DoA & FR & DR & DoR & VI \\
\hline $\begin{array}{l}\text { Protium heptaphyllum } \\
\text { (Aubl.) Marchand }\end{array}$ & 61 & 1,589 & 80,00 & 305,00 & 7,945 & 11,19 & 21,55 & 24,68 & 57,43 \\
\hline Eschweilera ovata (Cambess) Miers. & 59 & 0,643 & 100,00 & 295,00 & 3,216 & 13,99 & 20,85 & 9,99 & 44,83 \\
\hline Tapirira guianensis Aubl. & 7 & 0,662 & 25,00 & 35,00 & 3,309 & 3,50 & 2,47 & 10,28 & 16,25 \\
\hline Ocotea notata (Nees) Mez & 16 & 0,323 & 35,00 & 80,00 & 1,615 & 4,90 & 5,65 & 5,02 & 15,57 \\
\hline $\begin{array}{l}\text { Gochnatia polymorpha } \\
\text { (Less.) Cabrera. }\end{array}$ & 12 & 0,391 & 35,00 & 60,00 & 1,956 & 4,90 & 4,24 & 6,08 & 15,21 \\
\hline Pera glabrata Baill. & 9 & 0,441 & 30,00 & 45,00 & 2,206 & 4,20 & 3,18 & 6,85 & 14,23 \\
\hline Miconia prasina D.C. & 14 & 0,093 & 45,00 & 70,00 & 0,465 & 6,29 & 4,95 & 1,44 & 12,68 \\
\hline Myrcia fallax (Rich.)DC. & 12 & 0,220 & 35,00 & 60,00 & 1,097 & 4,90 & 4,24 & 3,41 & 12,55 \\
\hline $\begin{array}{l}\text { Pseudopiptadenia contorta (DC.) } \\
\text { G.P.Lewis \& M.P.M.de Lima }\end{array}$ & 11 & 0,260 & 25,00 & 55,00 & 1,298 & 3,50 & 3,89 & 4,03 & 11,42 \\
\hline Psychotria carthagenensis Jacq. & 6 & 0,060 & 25,00 & 30,00 & 0,299 & 3,50 & 2,12 & 0,93 & 6,55 \\
\hline Xylopia sericea A. St.-Hil. & 5 & 0,158 & 15,00 & 25,00 & 0,787 & 2,10 & 1,77 & 2,45 & 6,31 \\
\hline Byrsonima sericea DC. & 3 & 0,237 & 10,00 & 15,00 & 1,184 & 1,40 & 1,06 & 3,68 & 6,14 \\
\hline Guapira opposita (Vell.) Reitz & 5 & 0,104 & 15,00 & 25,00 & 0,518 & 2,10 & 1,77 & 1,61 & 5,47 \\
\hline $\begin{array}{l}\text { Eugenia punicifolia } \\
\text { (Humb., Bonpl. \& Kunth) DC. }\end{array}$ & 6 & 0,028 & 20,00 & 30,00 & 0,138 & 2,80 & 2,12 & 0,43 & 5,35 \\
\hline Sapindaceae & 7 & 0,051 & 10,00 & 35,00 & 0,257 & 1,40 & 2,47 & 0,80 & 4,67 \\
\hline Apuleia leiocarpa Vog. Macbride & 2 & 0,143 & 10,00 & 10,00 & 0,715 & 1,40 & 0,71 & 2,22 & 4,33 \\
\hline Licania sp. & 2 & 0,142 & 10,00 & 10,00 & 0,711 & 1,40 & 0,71 & 2,21 & 4,31 \\
\hline Inga hispida Schott ex Benth. & 6 & 0,020 & 10,00 & 30,00 & 0,102 & 1,40 & 2,12 & 0,32 & 3,84 \\
\hline Annona cacans Warming. & 3 & 0,035 & 15,00 & 15,00 & 0,173 & 2,10 & 1,06 & 0,54 & 3,69 \\
\hline $\begin{array}{l}\text { Rhodostemonodaphne } \\
\text { capixabensis Baitello \& Coe-Teixeira }\end{array}$ & 1 & 0,162 & 5,00 & 5,00 & 0,808 & 0,70 & 0,35 & 2,51 & 3,56 \\
\hline Calophyllum brasiliensis Cambess. & 1 & 0,156 & 5,00 & 5,00 & 0,780 & 0,70 & 0,35 & 2,42 & 3,48 \\
\hline Andira fraxinifolia Benth. & 3 & 0,013 & 15,00 & 15,00 & 0,067 & 2,10 & 1,06 & 0,21 & 3,37 \\
\hline $\begin{array}{l}\text { Miconia cinnamomifolia (DC.) } \\
\text { Naudin. }\end{array}$ & 2 & 0,077 & 10,00 & 10,00 & 0,383 & 1,40 & 0,71 & 1,19 & 3,29 \\
\hline $\begin{array}{l}\text { Inga flagelliformis (Vell.Conc.) } \\
\text { Martius. }\end{array}$ & 3 & 0,032 & 10,00 & 15,00 & 0,160 & 1,40 & 1,06 & 0,50 & 2,96 \\
\hline Inga laurina Willd. & 2 & 0,050 & 10,00 & 10,00 & 0,249 & 1,40 & 0,71 & 0,77 & 2,88 \\
\hline Solanum inaequale Vell. & 2 & 0,041 & 10,00 & 10,00 & 0,206 & 1,40 & 0,71 & 0,64 & 2,74 \\
\hline $\begin{array}{l}\text { Campomanesia guazumifolia } \\
\text { (Cambess.) O. Berg }\end{array}$ & 2 & 0,032 & 10,00 & 10,00 & 0,159 & 1,40 & 0,71 & 0,49 & 2,60 \\
\hline Ficus gomelleira Kunth \& Bouché & 1 & 0,094 & 5,00 & 5,00 & 0,471 & 0,70 & 0,35 & 1,46 & 2,52 \\
\hline Coccoloba alnifolia Casar. & 2 & 0,020 & 10,00 & 10,00 & 0,100 & 1,40 & 0,71 & 0,31 & 2,42 \\
\hline $\begin{array}{l}\text { Himatanthus phagedaenicus } \\
\text { (Mart.)Woodson }\end{array}$ & 2 & 0,018 & 10,00 & 10,00 & 0,088 & 1,40 & 0,71 & 0,27 & 2,38 \\
\hline Myrcia multiflora (Lam.) DC. & 3 & 0,011 & 5,00 & 15,00 & 0,054 & 0,70 & 1,06 & 0,17 & 1,93 \\
\hline Cupania zanthoxyloides Cambess. & 2 & 0,024 & 5,00 & 10,00 & 0,122 & 0,70 & 0,71 & 0,38 & 1,79 \\
\hline Sapium glandulatum (Vell.) Pax & 1 & 0,038 & 5,00 & 5,00 & 0,189 & 0,70 & 0,35 & 0,59 & 1,64 \\
\hline Myrsine guianensis (Aubl.) O.Kuntze & 1 & 0,014 & 5,00 & 5,00 & 0,070 & 0,70 & 0,35 & 0,22 & 1,27 \\
\hline Maytenus sp. & 1 & 0,012 & 5,00 & 5,00 & 0,059 & 0,70 & 0,35 & 0,18 & 1,23 \\
\hline Ficus clusiifolia Schott & 1 & 0,009 & 5,00 & 5,00 & 0,046 & 0,70 & 0,35 & 0,14 & 1,20 \\
\hline Matayba guianensis Aubl & 1 & 0,009 & 5,00 & 5,00 & 0,043 & 0,70 & 0,35 & 0,13 & 1,19 \\
\hline Indeterminada 2 & 1 & 0,007 & 5,00 & 5,00 & 0,036 & 0,70 & 0,35 & 0,11 & 1,16 \\
\hline Casearia commersoniana Cambess. & 1 & 0,007 & 5,00 & 5,00 & 0,034 & 0,70 & 0,35 & 0,10 & 1,16 \\
\hline Myrsine umbellata Mart. & 1 & 0,006 & 5,00 & 5,00 & 0,030 & 0,70 & 0,35 & 0,09 & 1,15 \\
\hline Vismia brasiliensis Choisy. & 1 & 0,004 & 5,00 & 5,00 & 0,020 & 0,70 & 0,35 & 0,06 & 1,12 \\
\hline Thyrsodium schomburgkianum Benth. & 1 & 0,002 & 5,00 & 5,00 & 0,010 & 0,70 & 0,35 & 0,03 & 1,08 \\
\hline Fabaceae 1 & 1 & 0,002 & 5,00 & 5,00 & 0,010 & 0,70 & 0,35 & 0,03 & 1,08 \\
\hline
\end{tabular}


Tabela 5 - Parâmetros fitossociológicos das espécies amostradas no estádio avançado da APA da Lagoa de Jacunem. NI = Número de indivíduos; AB = Área basal; FA = Frequência absoluta; DA = Densidade absoluta; DoA = Dominância absoluta; FR = Frequência relativa; DR = Densidade relativa; DoR = Dominância relativa; and VI = Valor de importância.

Table 5 - Phytosociological parameters of the species sampled in the advanced stage of Lagoa de Jacunem EPA. NI=number of individuals; $A B=$ basal area; $F A=$ Absolute frequence; $F R=$ Relative frequence; $D A=$ absolute density; $D R=$ Relative density; $D o A=A b s o l u t e$ dominance; $D o R=$ Relative dominance; and $V I=$ importance value.

\begin{tabular}{|c|c|c|c|c|c|c|c|c|c|}
\hline Espécie & NI & $\mathbf{A B}$ & FA & DA & DoA & FR & DR & DoR & VI \\
\hline Eschweilera ovata (Cambess) Miers. & 57 & 1,0960 & 90,00 & 285,00 & 5,48 & 12,33 & 22,44 & 24,52 & 59,29 \\
\hline Protium heptaphyllum (Aubl.) Marchand & 60 & 0,9858 & 95,00 & 300,00 & 4,93 & 13,01 & 23,62 & 22,06 & 58,69 \\
\hline Thyrsodium schomburgkianum Benth. & 18 & 0,2222 & 45,00 & 90,00 & 11,11 & 6,16 & 7,09 & 4,97 & 18,22 \\
\hline Miconia prasina D.C. & 15 & 0,0817 & 50,00 & 75,00 & 0,41 & 6,85 & 5,91 & 1,83 & 14,58 \\
\hline Myrcia fallax (Rich.)DC. & 10 & 0,1870 & 35,00 & 50,00 & 0,94 & 4,79 & 3,94 & 4,18 & 12,92 \\
\hline Tapirira guianensis Aubl. & 5 & 0,3233 & 20,00 & 25,00 & 1,62 & 2,74 & 1,97 & 7,23 & 11,94 \\
\hline Pseudopiptadenia contorta (DC.) & 2 & 0,3838 & 5,00 & 10,00 & 1,92 & 0,68 & 0,79 & 8,59 & 10,06 \\
\hline \multicolumn{10}{|l|}{ G.P.Lewis \& M.P.M.de Lima } \\
\hline Pera glabrata Baill. & 4 & 0,1000 & 15,00 & 20,00 & 0,50 & 2,05 & 1,57 & 2,24 & 5,87 \\
\hline Andira fraxinifolia Benth. & 3 & 0,0947 & 15,00 & 15,00 & 0,47 & 2,05 & 1,18 & 2,12 & 5,35 \\
\hline Eugenia rotundifolia Casar. & 4 & 0,0550 & 15,00 & 20,00 & 0,28 & 2,05 & 1,57 & 1,23 & 4,86 \\
\hline Erythroxylum sp. & 5 & 0,0303 & 15,00 & 25,00 & 0,15 & 2,05 & 1,97 & 0,68 & 4,70 \\
\hline Calophyllum brasiliensis Cambess. & 2 & 0,0998 & 10,00 & 10,00 & 0,50 & 1,37 & 0,79 & 2,23 & 4,39 \\
\hline Guapira opposita (Vell.) Reitz & 3 & 0,0452 & 15,00 & 15,00 & 0,23 & 2,05 & 1,18 & 1,01 & 4,25 \\
\hline Cordia sellowiana Cham. & 4 & 0,0184 & 15,00 & 20,00 & 0,09 & 2,05 & 1,57 & 0,41 & 4,04 \\
\hline Ficus clusiifolia Schott & 1 & 0,1284 & 5,00 & 5,00 & 0,64 & 0,68 & 0,39 & 2,87 & 3,95 \\
\hline Copaifera langsdorffi Desf. & 1 & 0,1165 & 5,00 & 5,00 & 0,58 & 0,68 & 0,39 & 2,61 & 3,69 \\
\hline Psychotria carthagenensis Jacq. & 3 & 0,0144 & 15,00 & 15,00 & 0,07 & 2,05 & 1,18 & 0,32 & 3,56 \\
\hline Dictyoloma vandellianum Adr. Juss. & 2 & 0,0571 & 10,00 & 10,00 & 0,29 & 1,37 & 0,79 & 1,28 & 3,44 \\
\hline Swartzia apetala Raddi & 3 & 0,0084 & 15,00 & 15,00 & 0,04 & 2,05 & 1,18 & 0,19 & 3,42 \\
\hline Himatanthus phagedaenicus (Mart.) & 2 & 0,0385 & 10,00 & 10,00 & 0,19 & 1,37 & 0,79 & 0,86 & 3,02 \\
\hline Woodson & & & & & & & & & \\
\hline Zanthoxylum rhoifolium Lam. & 2 & 0,0323 & 10,00 & 10,00 & 0,16 & 1,37 & 0,79 & 0,72 & 2,88 \\
\hline Chrysophyllum lucentifolium Cronquist. & 2 & 0,0263 & 10,00 & 10,00 & 0,13 & 1,37 & 0,79 & 0,59 & 2,75 \\
\hline Sapindaceae & 2 & 0,0222 & 10,00 & 10,00 & 0,11 & 1,37 & 0,79 & 0,50 & 2,65 \\
\hline $\begin{array}{l}\text { Garcinia gardneriana } \\
\text { (Planch. \& Triana) Zappi. }\end{array}$ & 2 & 0,0172 & 10,00 & 10,00 & 0,09 & 1,37 & 0,79 & 0,38 & 2,54 \\
\hline Ocotea sp. & 2 & 0,0158 & 10,00 & 10,00 & 0,08 & 1,37 & 0,79 & 0,35 & 2,51 \\
\hline Cupania zanthoxyloides Cambess. & 2 & 0,0111 & 10,00 & 10,00 & 0,06 & 1,37 & 0,79 & 0,25 & 2,41 \\
\hline Myrsine umbellata Mart. & 2 & 0,0100 & 10,00 & 10,00 & 0,05 & 1,37 & 0,79 & 0,22 & 2,38 \\
\hline Myrcia racemosa (O. Berg) Kiaersk. & 2 & 0,0054 & 10,00 & 10,00 & 0,03 & 1,37 & 0,79 & 0,12 & 2,28 \\
\hline \multicolumn{10}{|l|}{ Bonpl. \& Kunth) DC. } \\
\hline Fabaceae 2 & 1 & 0,0368 & 5,00 & 5,00 & 0,18 & 0,68 & 0,39 & 0,82 & 1,90 \\
\hline Cybistax antisyphilitica Mart. & 2 & 0,0137 & 5,00 & 10,00 & 0,07 & 0,68 & 0,79 & 0,31 & 1,78 \\
\hline Chamaecrista ensiformis (Vell.) & 2 & 0,0112 & 5,00 & 10,00 & 0,06 & 0,68 & 0,79 & 0,25 & 1,72 \\
\hline \multicolumn{10}{|l|}{ H. S. Irwin \& Barneby } \\
\hline Inga flagelliformis (Vell.Conc.)Martius. & 1 & 0,0256 & 5,00 & 5,00 & 0,13 & 0,68 & 0,39 & 0,57 & 1,65 \\
\hline Inga subnuda Salzm. ex Benth. & 1 & 0,0219 & 5,00 & 5,00 & 0,11 & 0,68 & 0,39 & 0,49 & 1,57 \\
\hline Pterocarpus rohrii Vahl & 2 & 0,0042 & 5,00 & 10,00 & 0,02 & 0,68 & 0,79 & 0,09 & 1,57 \\
\hline Fabaceae 3 & 1 & 0,0154 & 5,00 & 5,00 & 0,08 & 0,68 & 0,39 & 0,34 & 1,42 \\
\hline Myrcia multiflora (Lam.) DC. & 1 & 0,0082 & 5,00 & 5,00 & 0,04 & 0,68 & 0,39 & 0,18 & 1,26 \\
\hline $\begin{array}{l}\text { Astrocaryum aculeatissimum } \\
\text { (Schott) Burret }\end{array}$ & 1 & 0,0077 & 5,00 & 5,00 & 0,04 & 0,68 & 0,39 & 0,17 & 1,25 \\
\hline Cecropia pachystachya Trecul & 1 & 0,0072 & 5,00 & 5,00 & 0,04 & 0,68 & 0,39 & 0,16 & 1,24 \\
\hline Solanum alatirameum Bitter & 1 & 0,0067 & 5,00 & 5,00 & 0,03 & 0,68 & 0,39 & 0,15 & 1,23 \\
\hline Carpotroche brasiliensis (Raddi) A.Gray & 1 & 0,0059 & 5,00 & 5,00 & 0,03 & 0,68 & 0,39 & 0,13 & 1,21 \\
\hline Macoubea guianensis Aubl. & 1 & 0,0060 & 5,00 & 5,00 & 0,03 & 0,68 & 0,39 & 0,13 & 1,21 \\
\hline Licania sp & 1 & 0,0056 & 5,00 & 5,00 & 0,03 & 0,68 & 0,39 & 0,13 & 1,20 \\
\hline Cupania cf scrobiculata L.C.Rich. & 1 & 0,0050 & 5,00 & 5,00 & 0,03 & 0,68 & 0,39 & 0,11 & 1,19 \\
\hline Gochnatia polymorpha (Less.) Cabrera. & 1 & 0,0052 & 5,00 & 5,00 & 0,03 & 0,68 & 0,39 & 0,12 & 1,19 \\
\hline Ocotea argentea Mez. & 1 & 0,0042 & 5,00 & 5,00 & 0,02 & 0,68 & 0,39 & 0,09 & 1,17 \\
\hline Annona cacans Warming. & 1 & 0,0035 & 5,00 & 5,00 & 0,02 & 0,68 & 0,39 & 0,08 & 1,16 \\
\hline Gomidesia martiana O. Berg & 1 & 0,0037 & 5,00 & 5,00 & 0,02 & 0,68 & 0,39 & 0,08 & 1,16 \\
\hline Lauraceae 1 & 1 & 0,0030 & 5,00 & 5,00 & 0,02 & 0,68 & 0,39 & 0,07 & 1,15 \\
\hline Miconia sp. & 1 & 0,0032 & 5,00 & 5,00 & 0,02 & 0,68 & 0,39 & 0,07 & 1,15 \\
\hline Casearia commersoniana Cambess. & 1 & 0,0027 & 5,00 & 5,00 & 0,01 & 0,68 & 0,39 & 0,06 & 1,14 \\
\hline Inga laurina Willd. & 1 & 0,0023 & 5,00 & 5,00 & 0,01 & 0,68 & 0,39 & 0,05 & 1,13 \\
\hline Xylopia sericea A. St.-Hil. & 1 & 0,0023 & 5,00 & 5,00 & 0,01 & 0,68 & 0,39 & 0,05 & 1,13 \\
\hline Couepia schottii Fritsch & 1 & 0,0020 & 5,00 & 5,00 & 0,01 & 0,68 & 0,39 & 0,05 & 1,12 \\
\hline Lauraceae 2 & 1 & 0,0018 & 5,00 & 5,00 & 0,01 & 0,68 & 0,39 & 0,04 & 1,12 \\
\hline
\end{tabular}

Revista Árvore, Viçosa-MG, v.35, n.3, p.445-456, 2011 


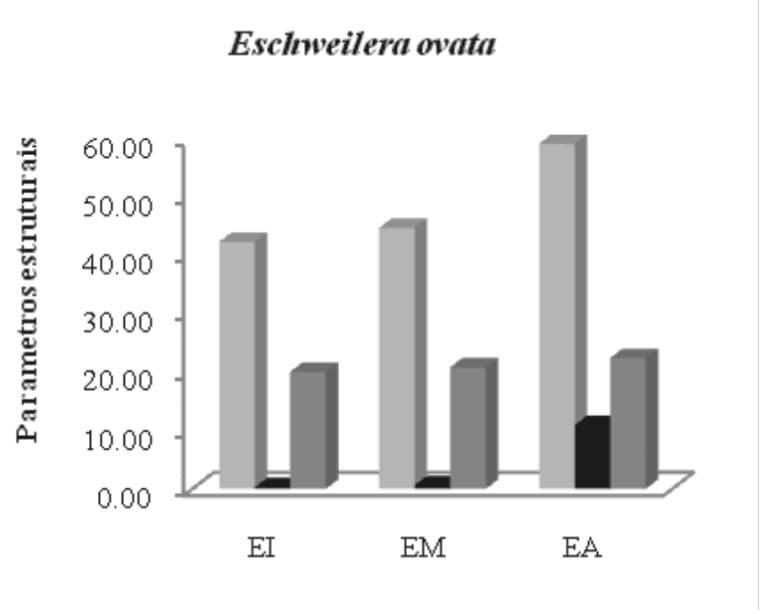

Protium heptapliyllum

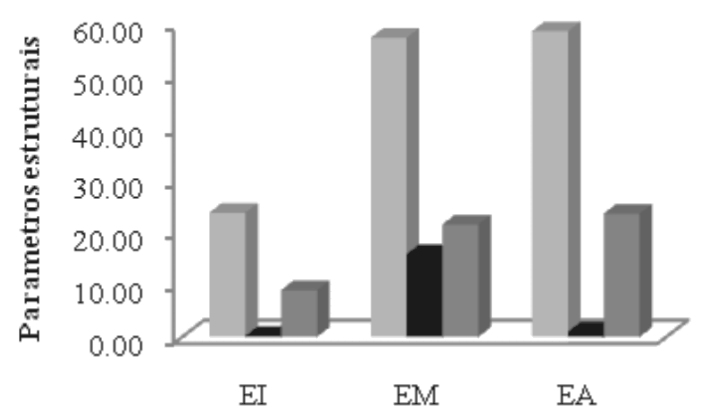

Myrcia fallax

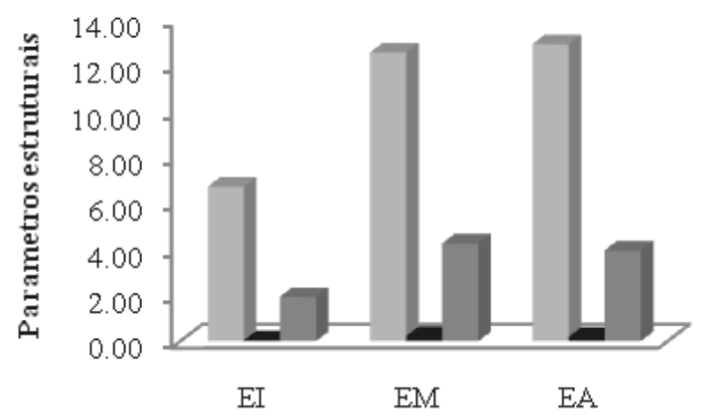

Gochnatia polymorpha

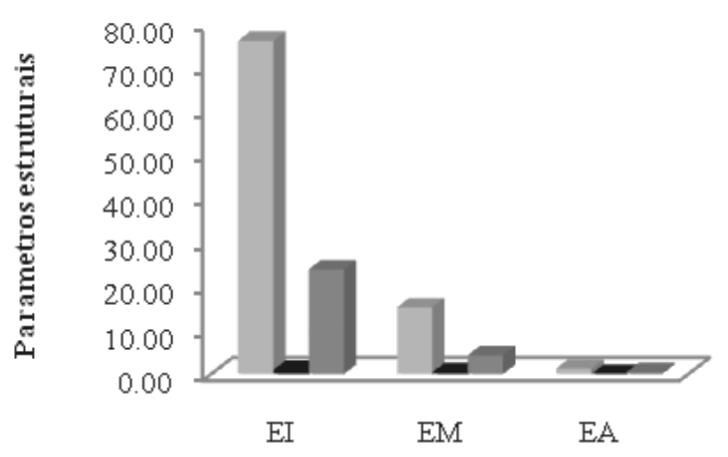

Tapirira guianensis

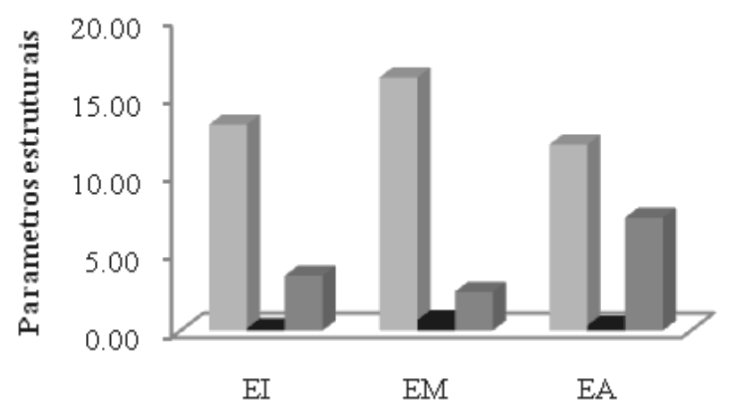

Pera glabrata

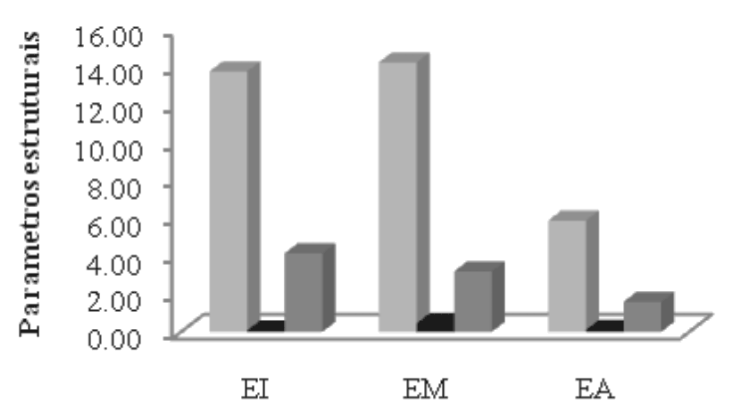

Figura 3 - Análise dos parâmetros estruturais de seis espécies presentes nos três estádios sucessionais analisados. EI Estádio inicial; EM = Estádio médio; EA = Estádio avançado de sucessão; $\quad$ V = Valor de importância (VI); $\square$ = Área basal (AB); = Densidade relativa (DR).

Figure 3 - Analysis of the structural parameters of six species in three analyzed succession stages. EI=Initial stage; EM = Middle stage; EA=Advanced stage; = Importance value (VI); = Relative density (DR); and = Basal area (AB). 
vegetação, tendo também a importante função de garantir a resiliência das florestas mais avançadas sucessionalmente.

O aumento da diversidade encontrado a partir dos estádios iniciais para o médio e avançado revela o avanço sucessional de formações florestais (TABARELLI; MANTOVANI, 1999; CAVALCANTE et al., 2000; OLIVEIRA et al., 2006). As mudanças microclimáticas geradas pelo maior sombreamento da vegetação, à medida que avançam os processos sucessionais, provavelmente permitem que maior número de espécies se estabeleçam.

O maior desenvolvimento da área basal encontrado no estádio médio contrasta com a definição apresentada pela Lei N 5.361 de Política Florestal do Estado do Espírito Santo, que define como estádio médio de regeneração uma área de floresta que apresenta área basal compreendida entre 10 e a menor que $18 \mathrm{~m}^{2} /$ ha. A classificação em estádio médio se deu por características estruturais observadas, presença de espécies pioneiras como Gochnatia polymorpha entre as dez mais importantes (VI), desta forma, se diferindo-se do trecho considerado avançado. As áreas basais encontradas nos estádios considerados avançado e inicial na APA Lagoa de Jacunem enquadram-se dentro da classificação prevista pela referida Lei. Esses dados indicam que somente a análise da área basal dos remanescentes florestais não é suficiente para considerar o avanço sucessional, já que este pode ser mascarado pela presença de indivíduos que apresentam grandes diâmetros, que, em sua maioria, devem ser remanescentes do corte seletivo.

A gradual redução no valor de importância, da área basal e densidade relativa de Gochnatia polymorpha, em direção aos estádios serais mais avançados, demonstra o avanço sucessional nos trechos considerados em estádio médio e avançado, já que esta espécie é tipicamente pioneira. Esta é citada em alguns estudos entre as de maior valor de importância em florestas em estádios iniciais de regeneração (CARDOSO-LEITE et al., 2004; SANTANA et al., 2004), tendo alta predominância fitofisionômica em áreas neste estádio sucessional (LIMA et al., 2006).

Eschweilera ovata é classificada por Jesus e Rolim (2005) para as florestas de Tabuleiro do norte do Espírito Santo como secundária tardia, sendo encontrada por Oliveira et al. (2006) restrita aos trechos de florestas com maior avanço sucessional. Desse modo, a presença desta espécie entre as mais importantes nos três estádios sucessionais analisados indicam o potencial de avanço sucessional das florestas ciliares da Lagoa Jacunem, sendo interessante ressaltar que o trecho em estágio inicial já apresenta capacidade de abrigar espécies mais exigentes quanto à disponibilidade de luz. O alto desenvolvimento estrutural de Pera glabrata e Tapirira guianensis contribuiu para caracterização do trecho em estádio médio de sucessão.

A maior representatividade estrutural dos indivíduos mortos em pé no estádio médio e o decréscimo delas para o estádio avançado indicam a substituição das espécies pioneiras do estádio inicial, sendo esta condição um resultado do avanço no processo sucessional. A mortalidade de espécies pioneiras à medida que segue o avanço na sucessão faz parte da dinâmica sucessional das florestas (LAURENCE et al., 1998). Isso se explica pelo tempo de vida relativamente curto da maioria das espécies pioneiras, normalmente de 10 anos (FERRETTI et al., 1995; MARTINS, 2007), raramente atingindo duas décadas.

Pode-se constatar que as características estruturais, diversidade e espécies dominantes presentes em cada estádio sucessional florestal, foram uma ferramenta eficaz indicativa do avanço sucessional das florestas ciliares da APA Lagoa de Jacunem. Usadas em conjunto, são eficientes para a classificação fisionômica de florestas.

Embora os resultados obtidos nas análises de solo não tenham sido suficientes para esclarecimento do avanço sucessional das florestas, isso é relevante no que tange à indicação de espécies para projetos de restauração florestal, haja vista, que as espécies com maior valor de importância deste estudo podem ser utilizadas para a recuperação de áreas degradadas onde os solos são pobres em nutrientes e com acidez e alumínio elevados.

\section{AGRADECIMENTOS}

Às empresas Makoto/Associação das Empresas de Mármore e Granito da Serra/Sindirochas, pelo financiamento deste estudo; a Thiago S. Soares, pela elaboração do abstract; a Mariana F. Rocha, pelo auxílio nas atividades de campo; e a Flavia P. Garcia, pelo auxílio na determinação das espécies de Fabaceae. 


\section{REFERÊNCIAS}

ALVAREZ V., V. H. et al. Interpretação dos resultados das análises de solos. In: RIBEIRO, A C.; GUIMARÃES, P. T. G.; ALVAREZ V., V. H.. (Eds.) Recomendações para uso de corretivos e fertilizantes em Minas Gerais. Viçosa, MG: CFSEMG, 1999. p. 25-32.

ARAÚJO, F. S. et al. Estrutura da vegetação arbustiva-arbórea colonizadora de uma área degradada por mineração de caulim, Brás Pires, MG. Revista Árvore, v.30, n.1, p.106-116, 2006.

ARAÚJO, M. M. et al. Padrão e processo sucessional em floresta secundária de diferentes idades na Amazônia Oriental. Ciência Florestal, v.15, n.4, p.343-357, 2005.

BROWER, J. E.; ZAR, J. H. Field and laboratory methods for general ecology. 2.ed. Dubuque: W. C. Brown Company, 1984. 226p.

BROWN, S.; LUGO, A. E. Tropical secondary forests. Journal of Tropical Ecology, n.6, p.1-32, 1990.

CARDOSO-LEITE, E. et al. Fitossociologia e caracterização sucessional de um fragmento de mata ciliar, em Rio Claro/SP, como subsídio à recuperação da área. Revista do Instituto Florestal, v.16, n.1, p.31-41, 2004.

CAVALCANTE, A. M. B.; SOARES, J. J.; FIGUEIREDO, M. A. Comparative phytosociology of tree sinusiae between contiguous forests in different stages of succession. Revista Brasileira Biologia, v.60, n.4, p.551-562, 2000.

FERRETTI, A. R. et al. Classificação das espécies arbóreas em grupos ecológicos para revegetação com nativas no Estado de São Paulo. Florestar Estatístico, v.3, n.7, p.73-77, 1995.

GUARIGUATA, M. R.; OSTERTAG, R. Neotropical secondary forest sucession: changes in structural and functional characteristics. Forest Ecology and Management, n.148, p.185-206, 2001.
FUNDAÇÃO INSTITUTO BRASILEIRO DE GEOGRAFIA E ESTATÍSTICA - IBGE. Folha SF. 34 Rio Doce: geologia, geomorfologia, pedologia, vegetação e uso potencial da terra. Rio de Janeiro: Projeto RADAMBRASIL, 1987.

JACOMINE, P. K. T. Solos sob matas ciliares. In: RODRIGUES, R. R.; LEITÃO-FILHO, H. F. (Eds.). Matas ciliares: conservação e recuperação. São Paulo: EDUSP/FAPESP, 2001. p.27-32.

JESUS, R. M.; ROLIM, S. G. Fitossociologia da Mata Atlântica de Tabuleiro. Boletim Técnico SIF, v.19, p.1-149, 2005.

LAURENCE, W. F. et al. Rain forest fragmentation and the dynamics of Amazonian tree communities. Ecology, v.76, n.6, p.2032-2040, 1998.

LIMA, H. C. et al. Caracterização fisionômicoflorística e mapeamento da vegetação da Reserva Biológica de Poço das Antas, Silva Jardim, Rio de Janeiro, Brasil. Rodriguésia, v.57, n.6, p.369389, 2006.

LÓPEZ, J. A. Caracterização fitossociológica e avaliação econômica de um fragmento de mata atlântica secundária, município de Linhares - ES. 1996. 71p. Dissertação (Mestrado em Ciência Florestal) - Universidade Federal de Viçosa, Viçosa, 1996.

MAGNAGO, L. F. S.; ASSIS, A. M.; FERNANDES, H. Q. B. Floresta Ombrófila densa submontana, montana e alto-montana. In: SIMONELLI, M.; FRAGA, C. N. Espécies da flora ameaçadas de extinção no estado do Espírito Santo. Vitória: IPEMA, 2007. p. 29-34.

MARTINS, S. V. Recuperação de matas ciliares. 2.ed. Viçosa, MG: Centro de Produções Técnicas, 2007. 255p.

MUELLER-DOMBOIS, D.; ELLENBERG, H. Aims and methods of vegetation ecology. New York: J. Wiley \& Sons, 1974.

OLIVEIRA FILHO, A. T. et al. Diversity and structure of the tree community of a fragment of tropical secondary forest of the Brazilian Atlantic Forest domain 15 and 40 years after logging. Revista Brasileira de Botânica, v.27, n.4, p.685-701, 2004.

Revista Árvore, Viçosa-MG, v.35, n.3, p.445-456, 2011 
OLIVEIRA, F. X.; ANDRADE, L. A.; FÉLIX, L. P. Comparações florísticas e estruturais entre comunidades de Floresta Ombrófila Aberta com diferentes idades, no Município de Areia, PB, Brasil. Acta Botânica Brasílica, v.20, n.4, p.861-873, 2006.

PAULA, A.; LOPES, W. P.; SILVA, A. F. Florística e estrutura de fragmentos florestais no entorno da lagoa Juparanã, Linhares, Espírito Santo, Brasil.

Boletim do Museu de Biologia Mello Leitão (Nova Série), n.26, p.5-23, 2009.

PAULA, A. et al. Sucessão ecológica da vegetação arbórea em uma floresta estacional semidecidual em Viçosa, MG, Brasil. Acta Botânica Brasílica, v.18 n.3, p.407-423, 2004.

PEIXOTO A. L.; GENTRY, A. Diversidade e composição florística da mata de tabuleiro na Reserva Florestal de Linhares (Espírito Santo, Brasil). Revista Brasileira de Botânica, v.13, p.19-25, 1990.

ROLIM, S. G.; NASCIMENTO, H. E. M. Análise da riqueza, diversidade e relação espécie-abundância de uma comunidade arbórea tropical em diferentes intensidades amostrais. Scientia Forestalis, v.52, p.7-16, 1997.

SANTANA, C. A. A.; LIMA, C. C. D.; MAGALHÃES, L. M. S. Estrutura horizontal e composição florística de três fragmentos secundários na cidade do Rio de Janeiro. Acta Scientiarum Biological Sciences, v.26, n.4, p.443-451, 2004.

Revista Árvore, Viçosa-MG, v.35, n.3, p.445-456, 2011
SHEPHERD, G. J. Fitopac 1. Manual do usuário. Campinas: UNICAMP, 1994.

SIMONELLI, M. et al Floristic composition and structure of the tree component of a muçununga forest in the Linhares Foret Reserve, Espírito Santo, Brazil. Memoirs of the New York Botanical Garden, v.100, p.351-370, 2008.

TABARELLI, M.; MANTOVANI, W. A regeneração de uma floresta tropical montana após corte e queima (São Paulo - Brasil).

Revista Brasileira de Biologia, v.59, n.2, p.239-251, 1999.

TABARELLI, M. et al. Desafios e oportunidades para a conservação da biodiversidade da Mata Atlântica brasileira. Megadiversidade, v.1, p.1-7, 2005.

THOMAZ, L. D. Florística e Fitossociologia da Floresta Atlântica na Estação Biológica de Santa Lúcia, Santa Teresa - ES. 1996. 321f. Tese (Doutorado em Ciências Biológicas) Universidade Estadual Paulista, Rio Claro, São Paulo, 1996.

TILMAN, D. Plant strategies and the dynamics and structure of plant communities. Princeton: Princeton University Press, 1988. 362p.

VILELA, E. A. et al. Espécies de mata ciliares com potencial para estudos de revegetação no Alto Rio Grande em Itutinga, Sul de Minas. Revista Árvore, v.17, n.2, p.117-128, 1993. 